\title{
Editorial: Active Experiments in Space: Past, Present, and Future
}

\author{
Gian Luca Delzanno ${ }^{1 *}$, Joseph E. Borovsky ${ }^{2}$ and Evgeny Mishin ${ }^{3}$ \\ ${ }^{1}$ T-5 Applied Mathematics and Plasma Physics Group, Los Alamos National Laboratory, Los Alamos, NM, United States, \\ 2 Space Science Institute, Boulder, CO, United States, ${ }^{3}$ Space Vehicles Directorate, Air Force Research Laboratory, \\ Albuquerque, NM, United States
}

Keywords: active space experiments, plasma physics, magnetospheres, ionosphere, laboratory astrophysics, space physics

\section{Editorial on the Research Topic}

\section{Active Experiments in Space: Past, Present, and Future}

Between 1958 and 1962 the United States and the Soviet Union performed several nuclear detonation tests in the atmosphere, including the Starfish Prime event which involved a $1.4 \mathrm{Mt}$ explosion at $400 \mathrm{~km}$ altitude over Johnston island on July 9, 1962 (Gombosi et al., 2017). These tests can be considered as the beginning of active experiments in space (i.e., experiments that deliberately perturb the local environment). They demonstrated the potential destructive power of high-altitude nuclear explosions, both in terms of the resulting electromagnetic pulse as well as for the creation of a potentially long-lasting artificial radiation belt from the radioactive fission debris. For instance, one of the unintended consequences of Starfish Prime was to cripple at least seven spacecraft in low-Earth orbit (LEO), about a third of the LEO spacecraft of the time (Gombosi et al., 2017).

At about the same time, the fundamental discovery of the Earth's radiation belts by Van Allen and his team (Van Allen and Frank, 1959 and references therein) indicated how harsh the space environment could be for spacecraft and astronauts as well as how little we knew about it. Following the impetus of the Space Age, active space experiments flourished with the goals of (1) probing basic plasma physics phenomena, (2) elucidating aspects of magnetospheric and ionospheric physics, and (3) understanding how to control the effects of the environment on space assets. Bombs, beams, heaters, releases, chemical dumps, plasma plumes, tethers, antennas, voltages are examples of active experiments spanning several decades of research.

Six decades later the US active space experiment program has changed dramatically. The number of space-based experiments has seen a steep decline, supplanted by ground-based experiments that study the heating and modification of the ionosphere induced by powerful transmitters, such as the facilities of the High-Frequency Active Auroral Research Program (HAARP) and at Arecibo. This decline can be attributed to several reasons, summarized by the fact that the "low-hanging fruits" had already been collected and much more is known today about the space environment, that space flight became more bureaucratic and more risk-adverse, and budgetary pressures (Delzanno and Borovsky, 2018).

Yet, there are many reasons to be optimistic about the future of active experiments in space. There are new scientific and national-security drivers that demand new active space experiments. One example involves magnetosphere-ionosphere coupling, where a high-power electron beam could be used for magnetic field line mapping and connect phenomena occurring in the distant magnetosphere with their image in the ionosphere (National Research Council, 2012). Another example concerns radiation belt remediation, where the fluxes of an artificial radiation belt created by a high-altitude nuclear explosion could be substantially reduced by spacebased injection of electromagnetic plasma waves with the objective of protecting critical space assets. Furthermore, there are new maturing technologies (metamaterials, compact relativistic 
accelerators, antennas constructed of superparamagnetic nanoparticles, cube-satellites, ...), ever better diagnostics and data-gathering capabilities, and new computational tools that can support the design and interpretation of active experiments like never before. Indeed, this optimism was conveyed by the 65 participants of the Workshop "Active Experiments in Space: Past, Present and Future" who gathered in Santa Fe, New Mexico, in September 2017 (see the link http://www.cvent.com/events/ active-experiments-in-space-past-present-and-future/eventsummary-73675ac6ba5745d48d181933c4783454. aspx?dvce $=1$ for a list of talks presented at the workshop) and is echoed in this Frontiers special issue with the same name.

This special issue was designed to connect the past, the present, and the future of active experiments in space and serve as a reference for the community. It involves several review articles of past active experiments discussing chemical releases (Haerendel) and diamagnetic cavities (Winske et al.), artificial aurora experiments (Mishin), the APEX electronbeam experiments (Prech et al.), and an overview of active experiments involving the Los Alamos National Laboratory (Pongratz). A review of more recent active experiments focusing on electromagnetic wave injection and wave-particle interaction physics is given by Gołkowski et al.. A review on the potential use of electron beams to solve outstanding problems in space physics is presented by Sanchez et al., while a review on the future of active experiments is presented by Borovsky and Delzanno. The special issue also contains several "Original Research" articles. A major focus is on the research associated with the use of high-power electron beams for space applications. These articles include a discussion of the development of new, compact, relativistic electron accelerators (Lewellen et al.), a tether-based spacecraft charging mitigation scheme (Marchand and Delzanno), the evolution of a relativistic electron beam for magnetic-field line mapping in near-Earth space (Powis et al.) and how magnetic-field-line curvature affects the ionospheric accessibility of the electron beam (Willard et al.), a method for measuring the local magnetic-fieldline curvature in the inner magnetosphere with a variableenergy electron beam (Willard et al.), and the atmospheric signatures created by relativistic electron beams (Marshall et al.). New ionospheric experiments involving very-longdistance propagation of high-frequency waves are discussed by

\section{REFERENCES}

Alfvén, H. (1970). Plasma Physics, Space Research and the Origin of the Solar System. Stockholm: Nobel lecture.

Delzanno, G. L., and Borovsky, J. E. (2018). Assessing the future of space-based experiments. Eos. 99. doi: 10.1029/2018EO 100867

Gombosi, T. I., Baker, D. N., Balogh, A., Erickson, P. J., Huba, J. D., and Lanzerotti, L. J. (2017). Anthropogenic space weather. Space Sci Rev. 212:985. doi: $10.1007 /$ s11214-017-0357-5

National Research Council. (2012). "Magnetosphere-to-ionosphere fieldline tracing technology, in Solar and Space Physics: A Science for a Technological Society," (Washington, DC: National Academies Press), 333.
Yampolski et al. A simulation study of the effect of plasma releases on the equatorial spread $\mathrm{F}$ is presented by Zawdie et al., while the use of plasma releases to enhance energetic neutral atom imaging is discussed by Scime and Keesee. Active experiments for planetary missions are discussed by Gilet et al. for active probes and by Voshchepynets et al. for sounding radar operations.

In the words of Nobel laureate Hannes Alfvén (Alfvén, 1970): "The center of gravity of the physical sciences is always moving. Every new discovery displaces the interest and the emphasis. Equally important is that new technological developments open new fields for scientific investigation. To a considerable extent the way science takes depends on the construction of new instruments as is evident from the history of science." This is certainly true for active experiments and an exciting new season of active experiments in space awaits. Ad maiora!

\section{AUTHOR CONTRIBUTIONS}

All authors listed have made a substantial, direct and intellectual contribution to the work, and approved it for publication.

\section{FUNDING}

GD was supported by the Laboratory Directed Research and Development program at Los Alamos National Laboratory (LANL) under project 20200073DR. LANL is operated by Triad National Security, LLC, for the National Nuclear Security Administration of U.S. Department of Energy (DOE) (Contract No. 89233218CNA000001). JB was supported by NASA Heliophysics LWS TRT program via grant NNX16AB75G, by the NSF GEM Program via award AGS-1502947, and by the NASA Heliophysics Guest Investigator Program via grant NNX14AC15G. EM was supported by the AIR Force Office of Scientific Research LRIR 19RVCOR038.

\section{ACKNOWLEDGMENTS}

The authors thank Guru Ganguli, Brian Gilchrist, Bob Marshall, Dennis Papadopoulos, Ennio Sanchez, and Erik Tejero for helpful conversations and in particular they thank Gerhard Haerendel.

Van Allen, J. A., and Frank, L. A. (1959). Radiation around the Earth to a radial distance of 107,400 km. Nature 183:430. doi: 10.1038/183430a0

Conflict of Interest: The authors declare that the research was conducted in the absence of any commercial or financial relationships that could be construed as a potential conflict of interest.

Copyright (C) 2020 Delzanno, Borovsky and Mishin. This is an open-access article distributed under the terms of the Creative Commons Attribution License (CC BY). The use, distribution or reproduction in other forums is permitted, provided the original author(s) and the copyright owner(s) are credited and that the original publication in this journal is cited, in accordance with accepted academic practice. No use, distribution or reproduction is permitted which does not comply with these terms. 